\title{
Herbal Medicines for Cold Hypersensitivity in the Hands and Feet: A Systematic Review and Meta-Analysis
}

\author{
Jun-Sang $\mathrm{Yu}, \mathrm{PhD}$, Dongnyung Lee, $\mathrm{PhD}^{2}$ Daesung Hyun, $\mathrm{MSc}^{3,4}$ and Sei-Jin Chang, $\mathrm{PhD}^{5,6}$
}

\begin{abstract}
Objectives: Cold hypersensitivity in the hands and feet (CHHF) and Raynaud's phenomenon (RP) are prevalent among Asian populations, especially among women, who exhibit a higher rate of cold hypersensitivity that may be associated with gynecological problems. In several countries, herbal medicine has effectively treated cold hypersensitivity symptoms. This systematic review and meta-analysis of the literature was undertaken to evaluate the efficacy of herbal medicine for the treatment of CHHF in adults.

Design: Through March 31, 2018, comprehensive databases were searched, including MEDLINE, EMBASE, Cochrane Library, Chinese Academic Journal, and Japanese National Institute of Informatics, to identify relevant studies and extract data.

Outcome measures: Primary: total effective rate (TER); secondary: skin temperature, peripheral blood flow, adverse events.

Results: Fourteen randomized controlled trials $(n=974)$ were included. Thirteen studies with dichotomous values showed a significant reduction in CHHF and RP (risk ratio 0.31, 0.24-0.40) when comparing herbal medicine with/without Western medicine, and no treatment or Western medicine alone. Reductions in CHHF and RP were also observed between herbal medicine plus Western medicine and Western medicine alone (risk ratio $0.45,0.24-0.86$ ), as well as between herbal medicine and Western medicine alone (risk ratio 0.30, 0.210.41 ). In the only study using a placebo arm, herbal medicine was found to be superior to placebo in increasing skin temperature and peripheral blood flow. Six participants exhibited minor adverse drug reactions. Herbal medicine showed a superior TER, especially when combined with Western medicine, to Western medicine alone or placebo. However, there was a high risk of bias within all studies.

Conclusion: Although herbal medicine shows potential to be a safe and effective treatment for CHHF and RP, the high risk of bias in all studies prevents definitive conclusions; thus, higher quality studies must be performed.
\end{abstract}

Keywords: cold hypersensitivity, coldness, Raynaud's phenomenon, herbal medicine, systematic review, meta-analysis

\footnotetext{
${ }^{1}$ Department of Sasang Constitutional Medicine, College of Korean Medicine, Sangji University, Wonju, Republic of Korea.

${ }^{2}$ Department of Gynecology Medicine, College of Korean Medicine, Semyung University, Chungju, Republic of Korea.

Departments of ${ }^{3}$ Preventive Medicine and ${ }^{4}$ Biostatistics and Computing, The Graduate School of Yonsei University, Wonju, Republic of Korea.

${ }^{5}$ Department of Preventive Medicine and ${ }^{6}$ Institute of Environmental \& Occupational Medicine, Wonju College of Medicine, Yonsei University, Wonju, Republic of Korea.

( ) Jun-Sang Yu et al. 2018; Published by Mary Ann Liebert, Inc. This Open Access article is distributed under the terms of the Creative Commons License (http://creativecommons.org/licenses/by/4.0), which permits unrestricted use, distribution, and reproduction in any medium, provided the original work is properly cited.
} 


\section{Introduction}

C OLD HYPERSENSITIVITY IN THE hands and feet (CHHF) is a common symptom that occurs in $\sim 20 \%-52 \%$ of the Eastern Asian population, particularly in women., Women have a higher risk of developing CHHF than men; notably, a Korean study researching twin genetics found that the ratio of women to men (who exhibit CHHF) is $3: 2 .^{3} \mathrm{Al}-$ though the research and reviews related to $\mathrm{CHHF}$ have been increasing, the definition of $\mathrm{CHHF}$ has not been well established yet. In a previous study, ${ }^{4}$ the notion that " $\mathrm{CHHF}$ is a sensation of coldness in the hands and feet in an environment not considered cold by unaffected people or having a heightened cold sensation in a relatively low temperature area" or "'answering 'cold' to both 'are your hands cold or warm?' and 'are your feet cold or warm?', has been accepted. CHHF is associated with Raynaud's phenomenon (RP), an episodic vasospastic disorder, as well as with gastric disorders ${ }^{4}$ and gynecological problems. ${ }^{1,2}$ Specifically, in women, CHHF has been associated with gynecological problems, such as infertility and dysmenorrhea. ${ }^{5-10}$ The pathophysiological mechanisms of CHHF or RP remain unclear. ${ }^{11}$

In conventional medicine, $\mathrm{CHHF}$ is the representative clinical symptom of RP; therefore, most patients with $\mathrm{CHHF}$ are prescribed antihypertensive drugs or vasodilators. However, in Eastern medicine, traditional Chinese medicine, traditional Korean medicine, and traditional Japanese medicine (Kampo medicine), coldness is viewed as the main pathogenic factor. In Yin-Yang theory, body temperature goes down as Yang energy is exhausted and Yin energy is replenished. Balancing the body's Yin and Yang energy with holistic and personalized approaches is the primary focus of CHHF treatment in Eastern and traditional medicine.

For more than 2000 years, herbal medicine has been used to regulate biological coldness in many countries; to the best of the authors' knowledge, no systematic review of the efficacy of herbal medicine for the treatment of $\mathrm{CHHF}$ has been conducted and published in English. Thus, a systematic review and meta-analysis of the literature were conducted to determine whether herbal medicine treatments are more effective in improving CHHF symptoms than other treatments or placebos.

\section{Materials and Methods}

\section{Study design}

The target participants were patients who exhibited either $\mathrm{CHHF}$ or primary RP, and who were receiving conventional medical treatment or no treatment. Patients with CHHF or primary RP were defined as those diagnosed mainly by their chief complaints, cold stress test, or thermography. CHHF is the manifestation of the symptoms, not a disease, so it has no unified code in the International Classification of Diseases.

Intervention was defined as herbal medicine treatments, either herbal medicine alone or herbal medicine plus Western medicine. Comparison was defined as no treatment, Western medicine, placebo, or herbal medicine.

\section{Data sources and search strategy}

Studies were selected after a comprehensive search of the following databases: MEDLINE, EMBASE, CIHAHL, the
Cochrane Library, China National Knowledge Infrastructure, Japanese National Institute of Informatics, and OASIS (Korea). The search spanned the time period from the inception of each database to March 31, 2018, and used specific search string combinations according to each database for subject limitations within the English, Chinese, Japanese, and Korean languages. Authors of the selected studies without raw data were contacted for the detailed raw data.

\section{Study selection and criteria}

The target studies were randomized controlled trials that involved patients with $\mathrm{CHHF}$ or RP, if they met the following criteria: (1) randomized controlled trials that evaluated the effects of herbal medicine on patients with $\mathrm{CHHF}$ and (2) studies that provided the total effective rate (TER) or a visual analog scale (VAS) after completion of planned treatment. The exclusion criteria were as follows: (1) studies without a control group; (2) nonhuman clinical studies; (3) nonrandomized controlled trials; (4) studies that investigated the combined effects of herbal medicine and other therapies; (5) studies that did not provide any values (e.g., TER or VAS); and (6) studies that investigated CHHF caused by secondary RP, or by a specific disease (e.g., hypothyroidism, stroke, tumor, or diabetic neuropathy).

\section{Data extraction}

Two evaluators independently extracted the data from the studies, and discrepancies were discussed and solved either by negotiation or by a third party. Primary outcomes included the TER, discomfort intensity (e.g., VAS), and a numerical rating scale; secondary outcomes included temperature differences in the hands and feet, peripheral blood flow, and adverse events. The experiment, control, population, and outcomes of each study are summarized in Table 1.

\section{Study quality assessment}

Two reviewers independently evaluated the study's methodological quality using the Cochrane Handbook for Systematic Review of Intervention Version 5.1.0. A quality assessment included the following seven items: random sequence generation (selection bias), allocation concealment (selection bias), blinding of participants and personnel (performance bias), blinding of outcome assessor (detection bias), incomplete outcome data (attrition bias), selective reporting (reporting bias), and other biases. Each assessment was categorized into three levels: low risk, unclear risk, and high risk. Then, the trials were categorized as having high risk of bias (at least one item had a high risk of bias), unclear risk of bias (at least one item had an unclear risk of bias), or low risk of bias (all the items had a low risk of bias).

\section{Data synthesis and analysis}

For conducting the meta-analysis, statistical heterogeneity among the included studies was assessed using the $I^{2}$ index before the meta-analysis. ${ }^{12}$ If the $I^{2}$ statistic was $<50 \%$, the heterogeneity was regarded as acceptable, and the fixedeffects model was adopted. If it was $>50 \%$, the randomeffects model was used, because statistical heterogeneity would be significant. The risk ratio was used for dichotomous 


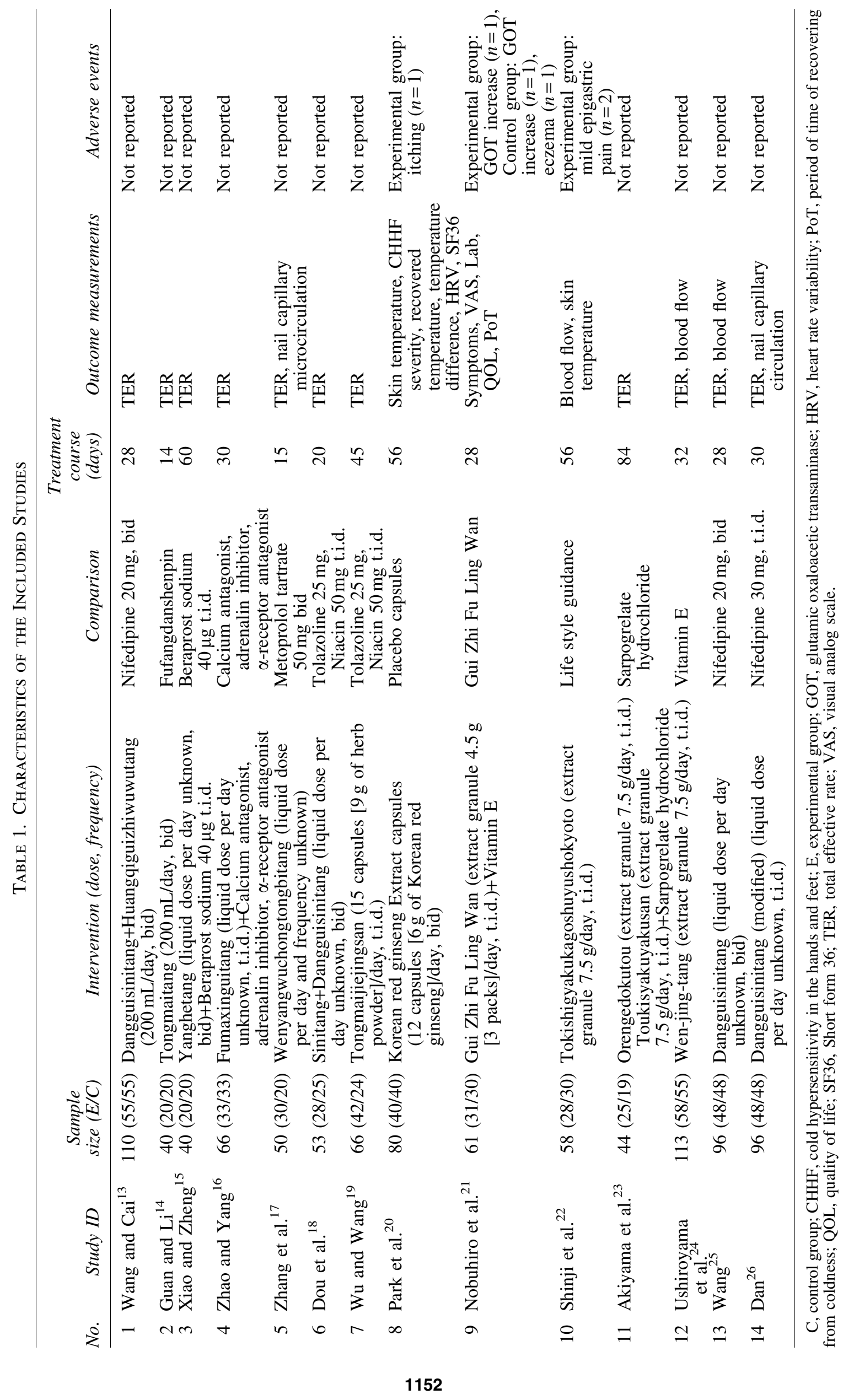



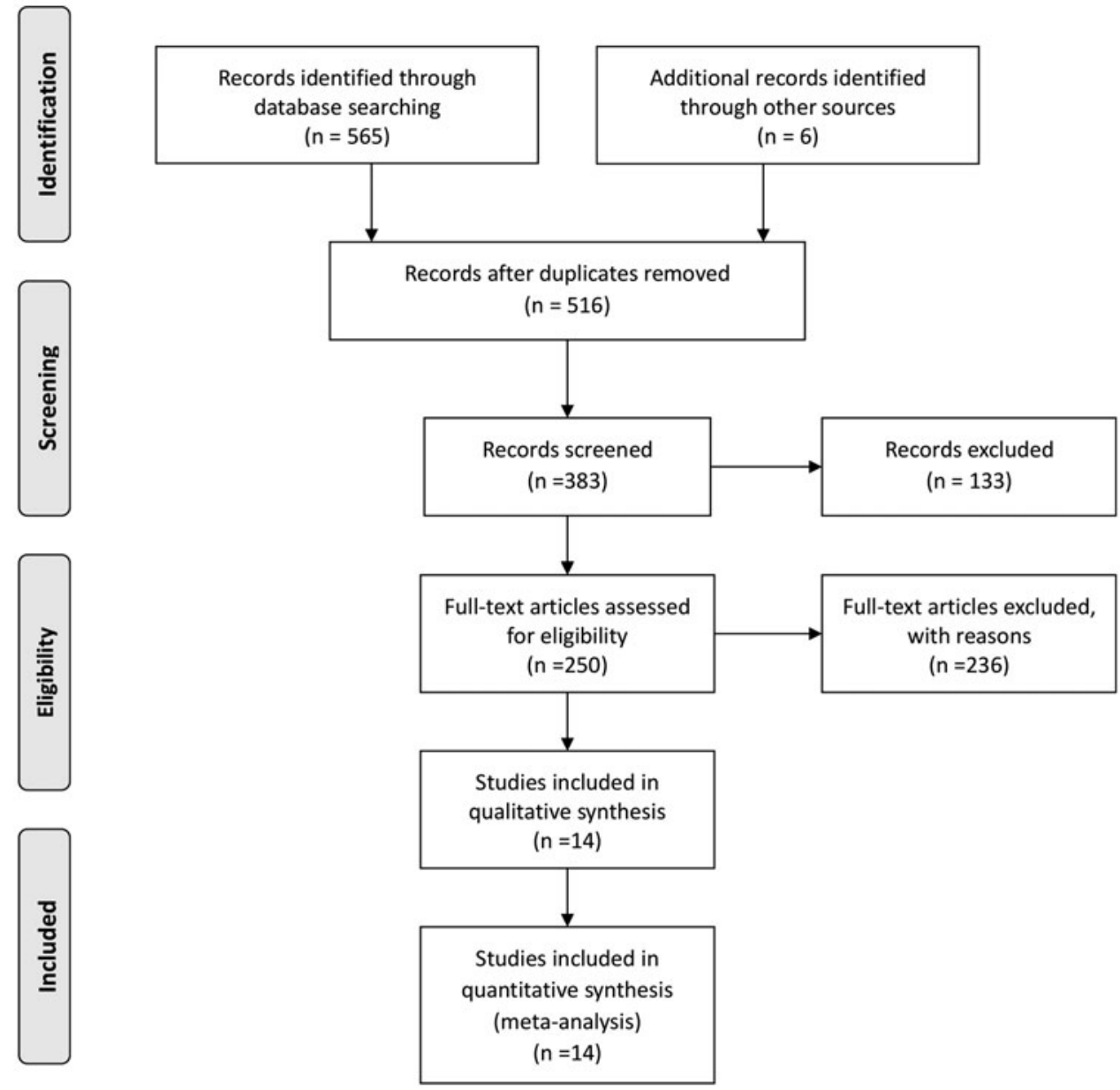

FIG. 1. PRISMA chart for cold hypersensitivity in the hands and feet.

outcomes, and the mean difference was used for continuous outcomes as effect estimates. RevMan5.3 (The Cochrane Collaboration, Oxford, United Kingdom) was used for meta-analysis. A funnel plot to check for the existence of publication bias might be performed, but in this study the number of included studies was small, and therefore, it was not executed.

\section{Outcome measurements}

The outcome was TER or VAS for symptom severity or temperature differences. The TER was evaluated using four items such as much improved, improved, not changed, and exacerbated. Much improved and improved were regarded as effective. The primary outcome comprised the TER or

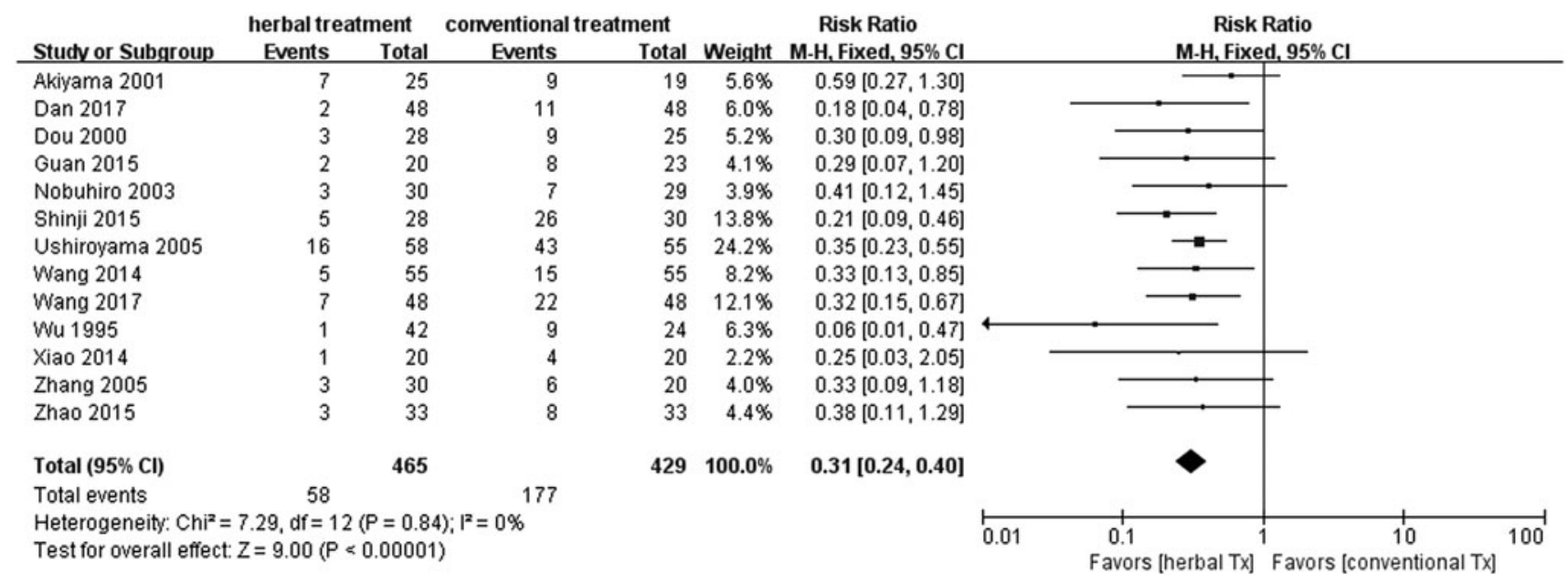

FIG. 2. Forest plot and risk of bias in the effect of herbal medicine treatment versus placebo or standard Western medications for $\mathrm{CHHF}$ using the fixed-effects model. $\mathrm{CHHF}$, cold hypersensitivity in the hands and feet. 


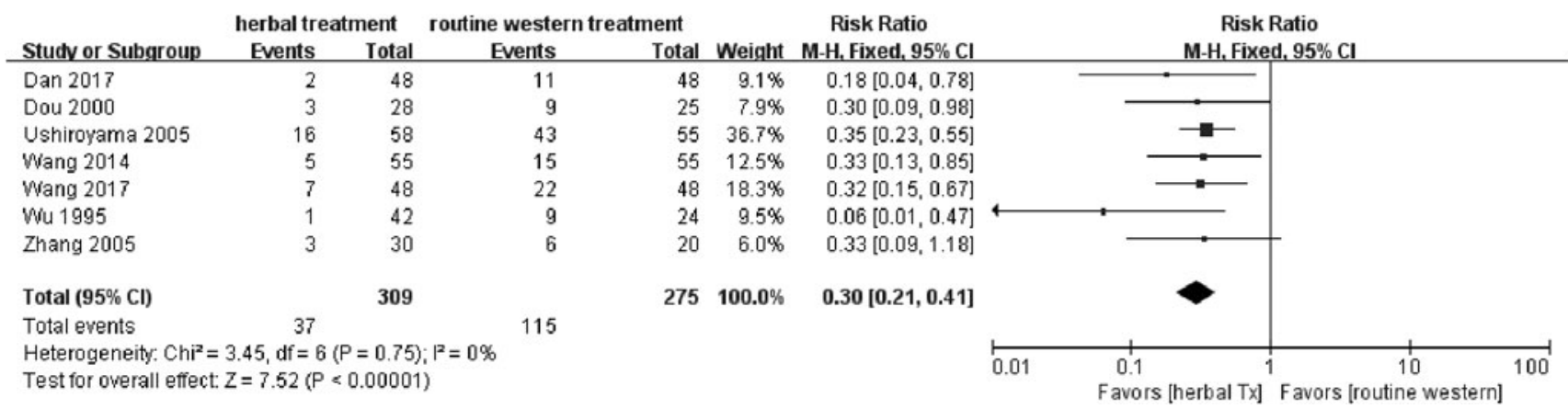

FIG. 3. Forest plot and risk of bias in the effect of herbal medicine treatment versus standard Western medications for CHHF using the fixed-effects model. CHHF, cold hypersensitivity in the hands and feet.

VAS; secondary outcomes were skin temperature, peripheral blood flow, and adverse events.

\section{Results}

\section{Study search results}

Through electronic searching, 571 records were identified. After the removal of duplicates $(n=55), 516$ records were screened. Initially, 383 studies met the inclusion criteria, and these studies then underwent full-text screening. In total, 14 randomized controlled trials were included. ${ }^{13-24}$ The study selection process is illustrated in Figure 1.

\section{Characteristics of the included studies}

A total of 974 participants in 14 trials, with sample sizes ranging from 19 to 58 subjects, were included in the analysis. Nine of the trials ${ }^{13-19,25,26}$ were conducted in China, one trial $^{20}$ was conducted in Korea, and four trials ${ }^{21-24}$ were conducted in Japan. Seven of the trials ${ }^{13,17-19,24-26}$ compared herbal medicine with standard Western medications. Three of the trials ${ }^{15,16,23}$ compared herbal medicine plus Western medications with Western medications. Two of the trials ${ }^{20,22}$ compared herbal medicine with placebo or no treatment. One of the trials ${ }^{21}$ compared herbal medicine plus Western medication with herbal medicine. One of the trials ${ }^{14} \mathrm{com}$ pared herbal medicine with a herbal medicine preparation (Fufangdanshenpin). The standard Western medications included nifedipine, beraprost sodium, adrenalin inhibitor, metoprolol tartrate, tolazoline, and sarpogrelate hydrochloride. The TER was measured in nine trials, ${ }^{13-19,23-26}$ skin temperature was measured in two trials, ${ }^{20,22}$ and peripheral blood flow by laser Doppler fluxmeter was measured in two trials. ${ }^{22,24-26}$ Herbal medicine treatment administration ranged from 2 to 12 weeks.

\section{Quality assessment of the included trials}

All the included trials mentioned randomization, but only seven of the trials ${ }^{13,14,19,20,22,25,26}$ described the method of randomization, including the random number table or randomization program. Only two trials ${ }^{20,22}$ mentioned a process for allocation concealment by an independent statistician through the end of the study. There was no information related to blind outcome assessments; therefore, all the trials were considered to have a high risk of bias.

\section{Outcomes of interventions}

Primary outcomes.

Total effective rate. Thirteen trials ${ }^{13-19,21,22,25,26}$ that measured the TER or VAS showed a significant difference in favor of herbal medicine with/without Western medicine compared with no treatment or Western medicine (risk ratio 0.31). Eleven trials ${ }^{13-19,23-26}$ measured TER. All nine Chinese studies and two of the Japanese studies included TER (Fig. 2). Seven trials ${ }^{13,17-19,24-26}$ compared herbal medicine treatment with standard Western medications and revealed a significant difference in favor of herbal medicine (risk ratio 0.30; Fig. 3); three trials ${ }^{15,16,23}$ compared herbal medicine treatment plus standard Western medications with standard Western medications alone. They also showed a significant difference in favor of herbal medicine plus standard Western medicine (risk ratio 0.45; Fig. 4). One tri$\mathrm{al}^{14}$ compared herbal medicine with a herbal medicine preparation (Fufangdanshenpin).

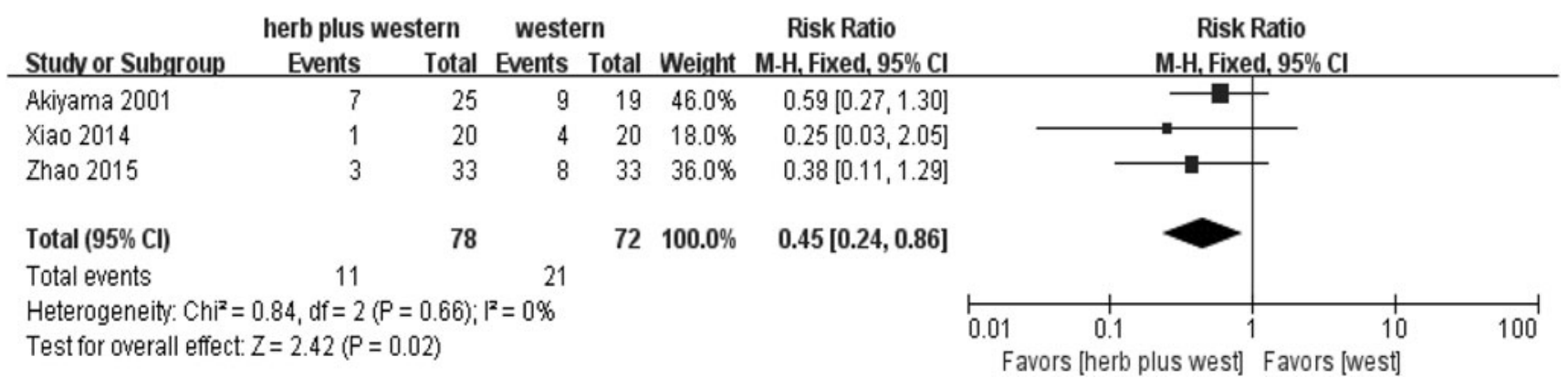

FIG. 4. Forest plot and risk of bias in herbal medicine treatment plus standard Western medications versus standard Western medications for CHHF using the fixed-effects model. CHHF, cold hypersensitivity in the hands and feet. 


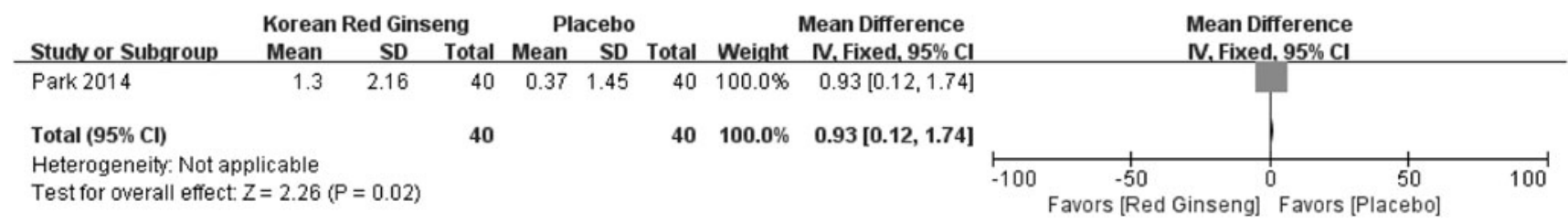

FIG. 5. Forest plot and risk of bias in herbal medicine treatment (Korean red ginseng) versus placebo for CHHF using the fixed-effects model. CHHF, cold hypersensitivity in the hands and feet.

Secondary outcomes.

Skin temperature. Two trials $\mathrm{s}^{20,22}$ evaluated outcomes using skin temperature. One trial ${ }^{22}$ detected no significance between the two forms of treatment, whereas the other tri$\mathrm{al}^{20}$ indicated that Korean red ginseng was more effective than placebo in reducing skin temperature differences between the palms and upper arms (mean difference $=0.93$, 95\% confidence interval $=0.12-1.74, p=0.02$; Fig. 5).

\section{Peripheral blood flow}

Four trials ${ }^{22,24-26}$ mentioned evaluating outcomes based on peripheral blood flow using a laser Doppler fluxmeter. First trial ${ }^{22}$ showed that tokishigyakukagoshuyushokyoto (TJ-38) was better than the control (life style guidance) for recovering blood flow $10 \mathrm{~min}$ after a 30 -s cold water test $(p=0.007)$. The second trial ${ }^{24}$ showed that wen-jing-tang (unkei-to) was better than the control (vitamin $\mathrm{E}$ administered) for increasing the peripheral surface blood flow of the tiptoe $(p=0.0068)$. Third trial ${ }^{25}$ showed that Danggui Sini Tang was better than Western medicine for changing the finger arterial blood flow peak in diastolic period. Fourth trial $^{26}$ showed that Danggui Sini Tang was better than Western medicine for improving nail capillary blood flow.

\section{Adverse events}

One trial ${ }^{21}$ reported that a participant in the experimental group (Guizhi fuling wang+vitamin E) and two participants in the control group (guizhi fuling wan) had adverse drug reactions of glutamic oxaloacetic transaminase increase and eczema. One trial ${ }^{20}$ reported that one participant in the experimental group (Korean red ginseng) presented with slight itching that disappeared after a few days. One trial ${ }^{22}$ reported that two participants in the experimental group (TJ-38) presented with a light stomachache. For these participants, the medication dosage was reduced by half, and the symptoms disappeared. The other nine trials ${ }^{13-19,23,24}$ did not mention any adverse drug reactions, and there were no serious adverse drug reactions reported in the 14 trials.

\section{Discussion}

A total of 14 studies (974 CHHF patients) were included in this review. From the results of the meta-analysis on 13 of the studies ${ }^{13-19,21-26}$ that had dichotomous values in terms of TER, a significant difference was observed between the herbal medicine group and the control group (relative risk 0.31 [0.24-0.40]; Fig. 2). The findings between the herbal medicine group and the Western medicine group ${ }^{13,17-20,24-26}$ were similar in all 13 studies $^{13-19,21-26}$ (relative risk 0.30 [0.21-0.41]; Fig. 3). When comparing herbal medicine plus

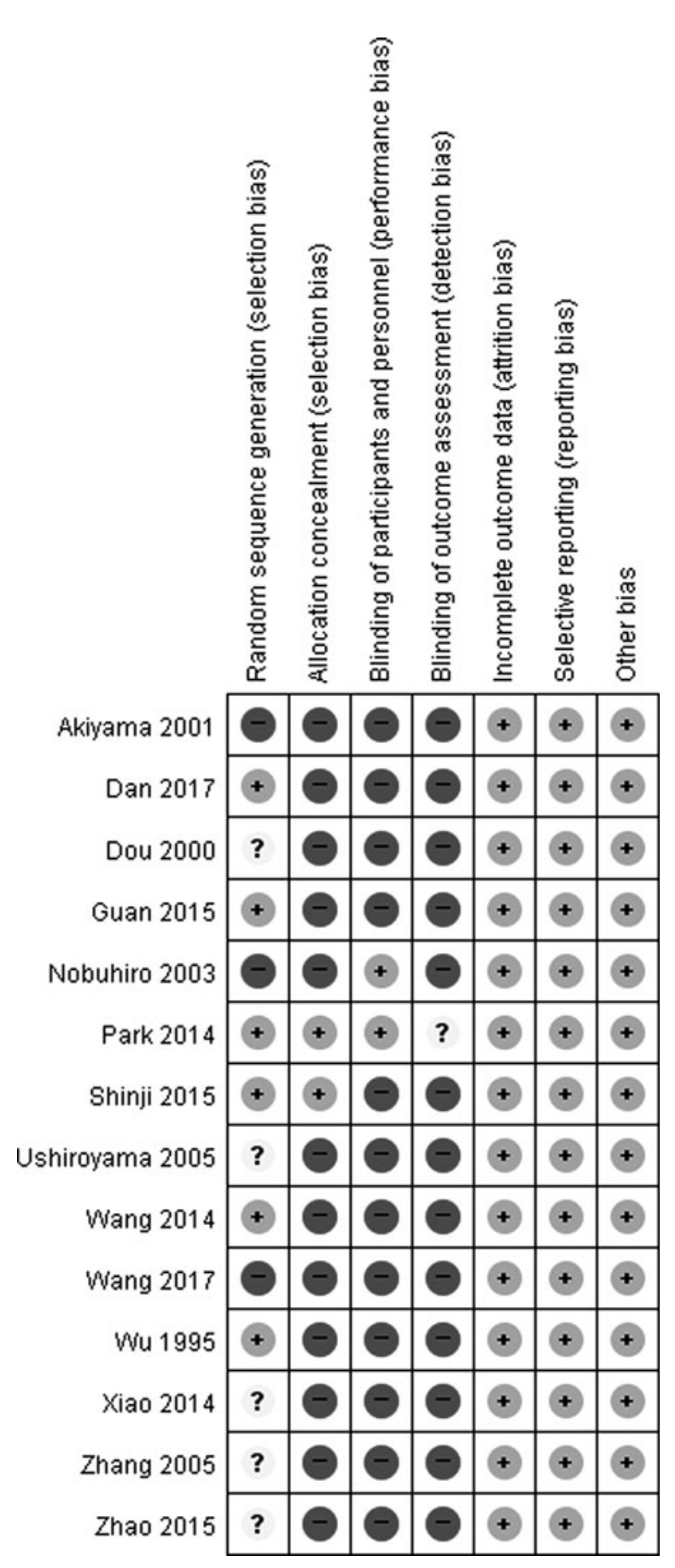

FIG. 6. Risk of bias assessment in the included studies. 


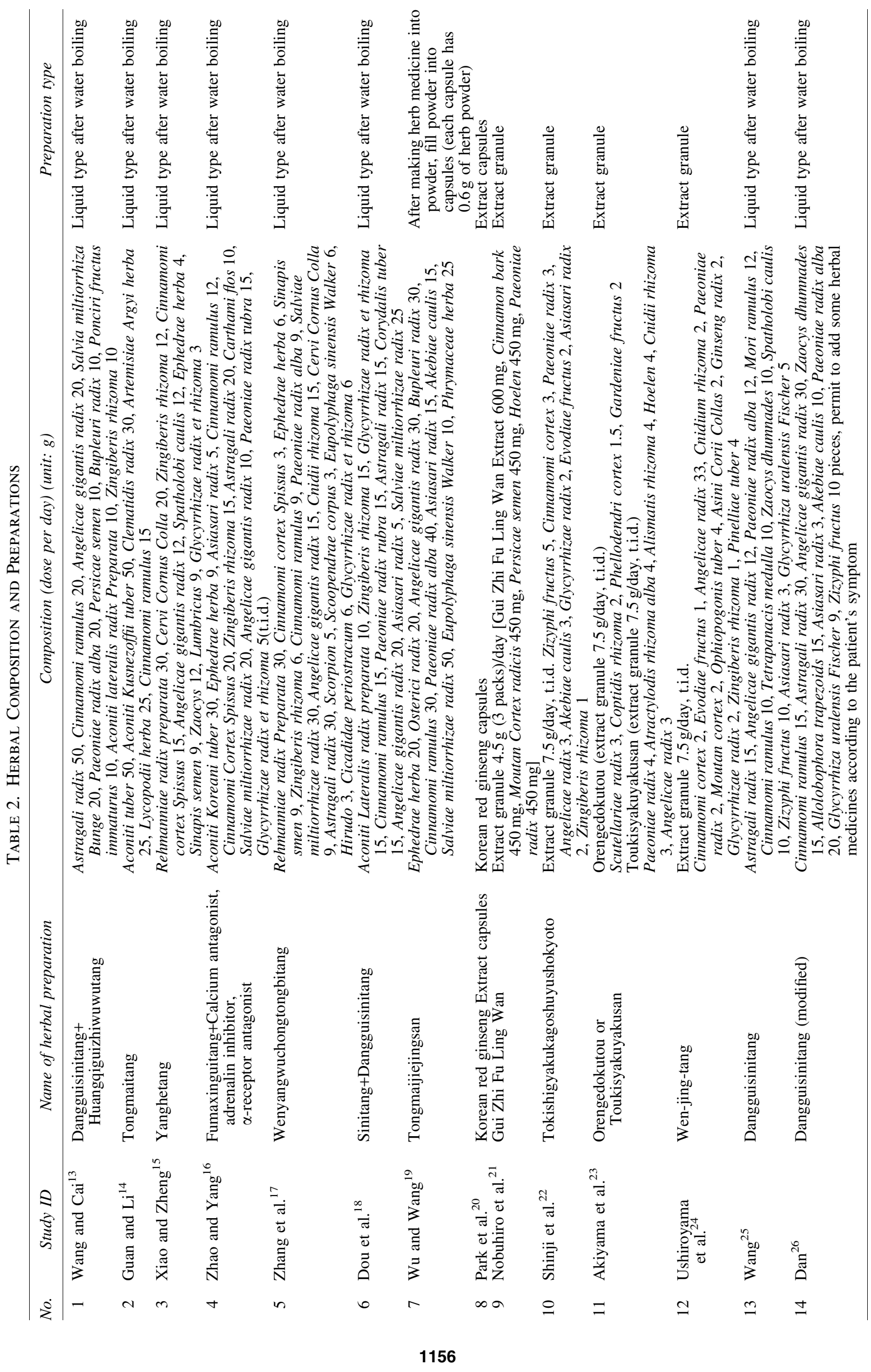


Western medicine with Western medicine alone, ${ }^{15,16,23}$ the results showed a significant difference (relative risk 0.45 [0.24-0.86]; Fig. 4). Consequently, although the results provide preliminary evidence that herbal medicine is a relatively safe and effective treatment for CHHF and RP, the high risk of bias in the existing studies makes ours a cautious conclusion. A significant improvement was observed in favor of the herbal medicine group in TER. One trial ${ }^{20}$ showed that Korean red ginseng has some therapeutic value for improving CHHF (Fig. 5), which had a high evidence level, but one of the participants taking the Korean red ginseng experienced itching as an adverse event. The overall quality of the 13 included studies suggested that there were considerable methodological problems of selection bias, performance bias, and detection bias (Fig. 6). ${ }^{13-19,21-26}$ All of the studies were small-population studies without sample size calculations. Nearly two-thirds of the included studies did not report on adverse events. ${ }^{13-19,23-26}$ Consequently, herbal medicine could be a safe and effective treatment for CHHF and RP, but the current evidence remains sparse due to risk of bias, requiring further studies on the subject.

Herbal preparations are shown in Table 2, which summarizes the contents of the herbal medicines according to dose per day. Analysis of the contents of the herbal medicines revealed that the most common herbal medicines were Cinnamomi ramulus or Cinnamomi cortex and Zingiberis rhizoma. Cinnamomi was present in 12 of 14 articles, and Zingiberis was present in 7 of 14 articles. These two herbal medicines have warm properties and a pungent smell in common.

Cinnamomi ramulus, or Cinnamomi cortex, being one of the most important spices worldwide, was traditionally administered for colds, flu, and digestive problems, and is still used in much the same way today. The key constituents are cinnamaldehyde, eugenol, tannins, coumarins, and mucilage. In both India and Europe, as well as East Asia, Cinnamomi has been traditionally taken as a warming herb for "cold" conditions, often in combination with Zingiberis. The herb stimulates the circulation, especially to the fingers and toes. It is also a traditional remedy for digestive problems, such as nausea, vomiting, and diarrhea, as well as for aching muscles and other symptoms of viral conditions, such as colds. ${ }^{27}$

Zingiberis is well researched; its key constituents are zingiberene, gingerol, and shogaols. Zingiberis was traditionally and is currently used to treat digestive problems, to serve as a circulatory stimulant, and to combat respiratory conditions. In traditional Eastern medicine, fresh Zingiberis is administered for fever, headache, and aching muscles, whereas dried Zingiberis is used for "internal cold" with symptoms such as cold hands, a weak pulse, and a pale complexion. ${ }^{27}$

There are strong points in this review. Primarily, to the authors' knowledge, this is the first review on the effects of herbal medicines used in the management of CHHF or RP. Herbal medicine is an important intervention in East Asian medicine, so the clinical evidence needed to be assessed. From this review, the authors tried to evaluate the clinical evidence, as well as the clinical application of herbal medicine. Second, an extensive search strategy was adopted without imposing any language limitations, including Chinese, English, Korean, and Japanese. Half of the included studies in this review were conducted in China and written in Chinese, which suggests that the strategy for the inclusion of the studies was appropriate. In this regard, studies of CHHF and RP were prevalent in East Asian countries, along with a high prevalence rate of CHHF. Unfortunately, a clear cause of this phenomenon has not been elucidated. It was assumed that the East Asian countries with high prevalence rates are located in the temperate climate region, and on islands or peninsulas. Third, the intervention details were summarized, including the herbal substance names provided in the prescriptions, the herbal medicine composition, the preparation type, and the dose frequency, and duration (Table 2). This information helps practitioners to apply these interventions in their own clinical practice.

This review also has limitations: The most important limitation originates from the included studies themselves, which are prone to serious risk of bias. Nearly two-thirds of the studies did not report on adverse events. In addition, most of the trials were published in China, which may indicate publication bias. Therefore, these risks of bias may impact the evidence to some extent. Second, the authors could not confirm whether all the included studies were really randomized controlled trials, because there is a report that found many clinical trials conducted in China could not be included in the authentic criteria of randomized controlled trials. ${ }^{28,29}$ A third limitation of the study is that dosage of the herbal intervention was not reported in 6 of 14 studies, and therefore those trials cannot be replicated.

On the basis of these results and in the near future, a randomized controlled trial of the herbal medicine extract was planned, using a double-blind method, to treat CHHF.

\section{Conclusion}

Although herbal medicine shows potential to serve as a safe and effective treatment for CHHF and RP, the high risk of bias in all studies prevents definitive conclusions; thus, higher quality studies must be performed.

\section{Acknowledgments}

This study was supported by the Traditional Korean Medicine R\&D Program funded by the Ministry of Health \& Welfare through the Korean Health Industry Development Institute (KHIDI; HB16C0048-010016).

\section{Author Disclosure Statement}

No competing financial interests exist.

\section{References}

1. Ushiroyama T, Kamimoto Y, Sakuma K, et al. Assessment of chilly sensation in Japanese women with laser Doppler fluxmetry and acceleration plethysmogram with respect to peripheral circulation. Bull Osaka Med Coll 2005;51: 76-84.

2. Lee SL, Lee KS, Song BK. The literature review on the cold hypersensitivity of women. Korean J Obstet Gynecol 1996;9:55-80.

3. Hur YM, Chae JH, Chung KW, et al. Feeling of cold hands and feet is a highly heritable phenotype. Twin Res Hum Genet 2012;15:166-169.

4. Bae KH, Lee JA, Park KH, et al. Cold hypersensitivity in the hands and feet may be associated with functional dys- 
pepsia: Results of a multicenter survey study. Evid Based Complement Alternat Med 2016;2016:1-8.

5. Sachiyo N, Shigeko H, Haruo Y. Relationship between pregnant women's sensitivity to cold(hiesho) and premature labor assessed using propensity scores for adjusting confounding factors. Jpn J Public Health 2012;59:381-389.

6. Sachiyo N, Shigeko H, Masako M. Inference of causal effects between pregnant women's sensitivity to cold(hiesho) and premature rupture of membranes-Adjustment of confounding factors by propensity scores. J Jpn Acad Midwif 2012;2692;190-200.

7. Sachiyo N, Shigeko H. Relationship between pregnant women's sensitivity to cold(hiesho) and abnormal delivery. J Jpn Acad Midwif 2013;27:94-99.

8. Sachiyo N, Shigeko H, Haruo Y. Estimation of causal effects of pregnant women's sensitivity to cold(hiesho) on uterine inertia and prolonged labor: Adjustment of confounding factors by propensity scores. J Jpn Acad Nurs Sci 2013;33:3-12.

9. Shirai M, Kuge H, Miyazaki J, et al. The relationship between a female hie(cold disorder) condition and infertility. J Jpn Soc Acupunct 2016;66:180-188.

10. Yuriko K, Yoko E. Relationships between cold sensitivity in pregnant women and perinatal outcomes. J Jpn Acad Midwif 2013;27:40-47.

11. Herrick AL. Pathogenesis of Raynaud's phenomenon. Rheumatology (Oxford) 2005;44:587-596.

12. Higgins JP, Thompson SG. Quantifying heterogeneity in a meta-analysis. Stat Med 2002;21:1539-1558.

13. Wang D, Cai ZM. Analysis of treatment effect of Dangguisinitang plus Huangqiguizhiwuwutang for Raynaud's syndrome. Contemp Diagn Treat 2014;18:4156-4157.

14. Guan L, Li GX. Tongmaitang oral in combination with Tongmaisan Fumigation treating Raynaud's disease random parallel control study. J Pract Trad Chinese Intern Med 2015;7:31-34.

15. Xiao L, Zheng GR. 20 cases of Raynaud's syndrome treated by Chinese and Western medicine combined therapy. Guangming Zhongyi 2014;7:1487-1488.

16. Zhao W, Yang Y. Clinical observation of 33 cases of Raynaud's syndrome treated by self made Fumaxinguitang. J Yunnan Zhongyi Zongyao 2015;1:49-50.

17. Zhang ZX, Zhang LJ, Zhang BT. 30 cases of Raynaud's syndrome treated by Onyangochungtongbitang. China Med Herald 2005;18:51.

18. Dou LG, Yu XH, Li YX, et al. Clinical observation of 28 cases for Raynaud's syndrome treated by Sinitang added prescription. Zhongyiyaoxuebao 2000;2:21.
19. Wu Y, Wang JX. 42 cases of Raynaud's syndrome treated by self made Tongmaijiejingsan. J New Chinese Med 1995;3:25-26.

20. Park KS, Park KI, Kim JW, et al. Efficacy and safety of Korean red ginseng for cold hypersensitivity in the hands and feet: A randomized, double-blind, placebo-controlled trial. J Ethnopharmacol 2014;158:25-32.

21. Nobuhiro S, Noriyuki $T$, Kenichi I, et al. Effects of a combination preparation of Guizhi Fuling Wand and vitamin $\mathrm{E}$ on indefinite complaints such as stiffness of shoulder and cold feeling. East Med 2003;19:23-43.

22. Shinji N, Eri E, Tetsuya O, et al. Effects of a traditional herbal medicine on peripheral blood flow in women experiencing peripheral coldness: A randomized controlled trial. BMC Complement Altern Med 2015;15:105.

23. Akiyama Y, Ohno S, Asaoka T, et al. The combination therapy with Sarpogrelate Hydrochloride and Kampo medicine (Oren-gedoku-to or Toki-shakuyaku-san) for Raynaud's phenomenon. Kampo Med 2001;51:1101-1108.

24. Ushiroyama T, Sakuma K, Nosaka S. Comparison of Effects of Vitamin E and Wen-jing-tang (Unkei-to), an herbal medicine, on peripheral blood flow in post-menopausal women with chilly sensation in the lower extremities: A randomized prospective study. Am J Chin Med 2006;34: 969-979.

25. Wang WD. Clinical observation of modified Danggui Sini Tang for Raynaud's disease. J New Chin Med 2017;49:44-45.

26. Dan MH. Clinical observation of modified Danggui Sini Tang for Raynaud's disease. Henan Med Res 2017;26: 1282-1283.

27. Chevallier A. Encyclopedia of Herbal Medicine. London, UK: Dorling Kindersley, 2000:155.

28. Wu T, Li L, Bian Z, et al. Randomized trials published in some Chinese journals: How many are randomized? Trails 2009; 10:46.

29. Purgato M, Cipriani A, Barbui C. Randomized trials published in Chinese or Western journals: Comparative empirical analysis. J Clin Psychopharmacol 2012;32:354-361.

Address correspondence to: Jun-Sang Yu, PhD

Department of Sasang Constitutional Medicine College of Korean Medicine Sangji University Wonju 26338 Republic of Korea

E-mail: hiruok@sangji.ac.kr 